\title{
Esophageal Candidiasis in a Patient With Uncontrolled Diabetes Mellitus and Recurrent Abdominal Pain, Symptomatic Or Incidental? A Case Report and Brief Literature Review
}

\author{
Nasir Saleem ${ }^{\mathrm{a}, \mathrm{b}}$, Saima Ijaz ${ }^{\mathrm{a}}$, Owais Lodhi ${ }^{\mathrm{a}}$
}

\begin{abstract}
Esophageal candidiasis (EC) is a common opportunistic infection in patients with impaired cellular immunity, such as human immunodeficiency virus (HIV) infection. However, it can also be found in individuals with certain predisposing conditions like uncontrolled diabetes mellitus (DM) and the use of certain medications. We report a 54-year-old female with past medical history of DM, hypertension and hyperlipidemia who presented to the ER with worsening periumbilical and epigastric pain for 2 days. She had multiple similar presentations requiring admissions to different hospitals and had undergone extensive workup over the past year. She only had transient improvement from these hospitalizations. Esophagogastroduodenoscopy (EGD) performed on day 2 of admission revealed extensive creamy pseudomembranous exudate through the entire length of esophagus confirmed as Candida albicans by brush scraping analysis. She received oral fluconazole with significant relief of her symptoms over the next couple of weeks. Although EC remains asymptomatic in the majority of patients and is only detected incidentally on EGD, its diagnosis and treatment can provide significant symptomatic relief in many patients. EC should be considered as a potential diagnosis in the appropriate clinical setting even in patients with no evidence of overt immunodeficiency.
\end{abstract}

Keywords: Esophageal candidiasis; Diabetes mellitus; Immunocompetent

\section{Introduction}

Esophageal candidiasis (EC) is one of the most common opportunistic infections in patients with impaired cellular immunity, such as human immunodeficiency virus (HIV) infection.

\section{Manuscript accepted for publication October 16, 2015}

aDepartment of Internal Medicine, Presence St. Joseph Hospital, 2900 N Lake Shore Dr., Chicago, IL 60657, USA

${ }^{b}$ Corresponding Author: Nasir Saleem, Department of Internal Medicine, Presence St. Joseph Hospital, 2900 N Lake Shore Dr., Chicago, IL 60657, USA. Email: nasirsaleemmd@gmail.com

doi: http://dx.doi.org/10.14740/jmc2337w
However, it can be found in individuals with no HIV infection and no evidence of opportunistic infections to suggest immunodeficiency. In such individuals, the presence of EC can usually be associated with other predisposing conditions. Such conditions include diabetes mellitus (DM), gastric acid suppression, chronic debilitating states like carcinomas and the use of systemic or inhaled corticosteroids, antibiotics and immunosuppressants. Patients with EC may remain asymptomatic or may present with one of the classic symptoms of infectious esophagitis, such as dysphagia, odynophagia and chest pain. Esophagogastroduodenoscopy (EGD) with brushings and biopsy remains the most sensitive and specific method for EC diagnosis. EC requires systemic antifungal therapy, and oral fluconazole is generally the recommended treatment due to its excellent efficacy, ease of administration and low cost. Although EC, in the majority of cases, is an incidental finding during EGD, its detection and treatment can provide significant symptomatic relief to patients.

\section{Case Report}

A 54-year-old female with past medical history of DM, hypertension and hyperlipidemia presented to the ER with worsening periumbilical and epigastric pain for 2 days. She denied dysphagia, odynophagia or melena but reported a weight loss of up to $20 \mathrm{lbs}$ over the past $6-8$ months. On physical exam she had a pulse rate of $86 \mathrm{bpm}, \mathrm{BP}$ of 170/80 and temperature of $98.1^{\circ} \mathrm{F}$. Her abdomen was soft, non-distended and mildly tender to palpation in the epigastrium and periumbilical region, but there was no rigidity, guarding or rebound tenderness. Bowel sounds were present and normal. Over the past 1 year she had multiple similar presentations with admissions to different hospitals and had undergone extensive workup with only transient improvement. Her last colonoscopy was 2 years ago and was reported as normal. On this admission her laboratory workup revealed a normal CBC, CMP, amylase, lipase and urinalysis. EKG did not reveal any signs of active cardiac ischemia. Her last $\mathrm{HbA1C}$ was $7.9 \%$, and she tested negative for HIV antibodies. Her X-ray KUB and CT abdomen revealed normal viscera and bowel gas pattern. She was given pain medications and intravenous fluids, and her symptoms improved substantially over the next 2 days. She underwent EGD on day 2 of admission which revealed extensive 


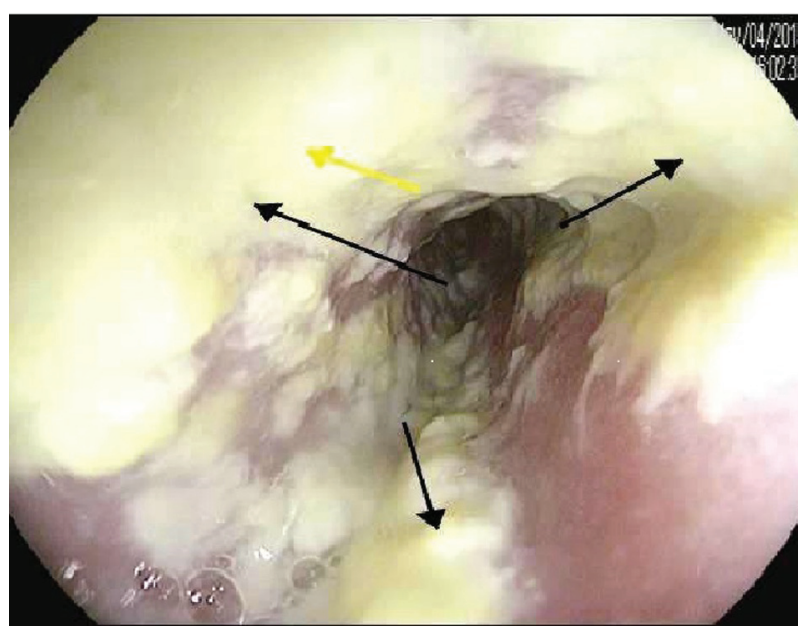

Figure 1. EGD: upper third of the esophagus showing creamy exudate (arrows).

creamy pseudomembranous exudate (Fig. 1-3) through the entire length of esophagus suspicious for EC. $150 \mathrm{~mL}$ of gastric fluid was also noticed in her stomach raising suspicion for gastroparesis given her history of DM; however, this was refuted by a nuclear medicine gastric emptying study done later during the admission. Brush scrapings obtained at the time of EGD confirmed the presence of pseudohyphae later identified as Candida albicans. She was started on fluconazole and discharged on day 3 of admission. Her symptoms improved to a great extent as noticed on a subsequent office visit with her primary care physician.

\section{Discussion}

$\mathrm{EC}$ is one of the most common opportunistic infections in patients with impaired cellular immunity, such as HIV infection [1]. However, it can be found in individuals with no HIV in-

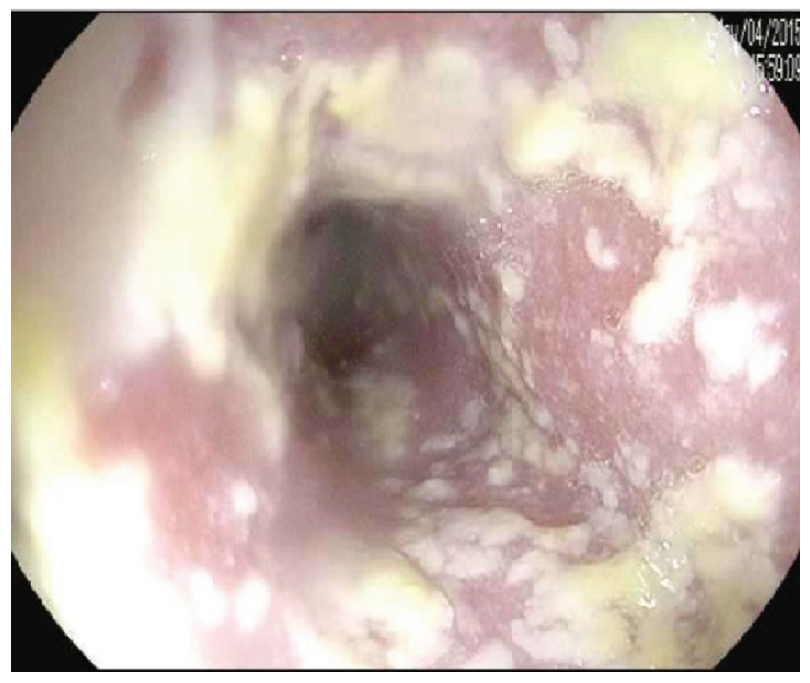

Figure 2. EGD: middle third of the esophagus.

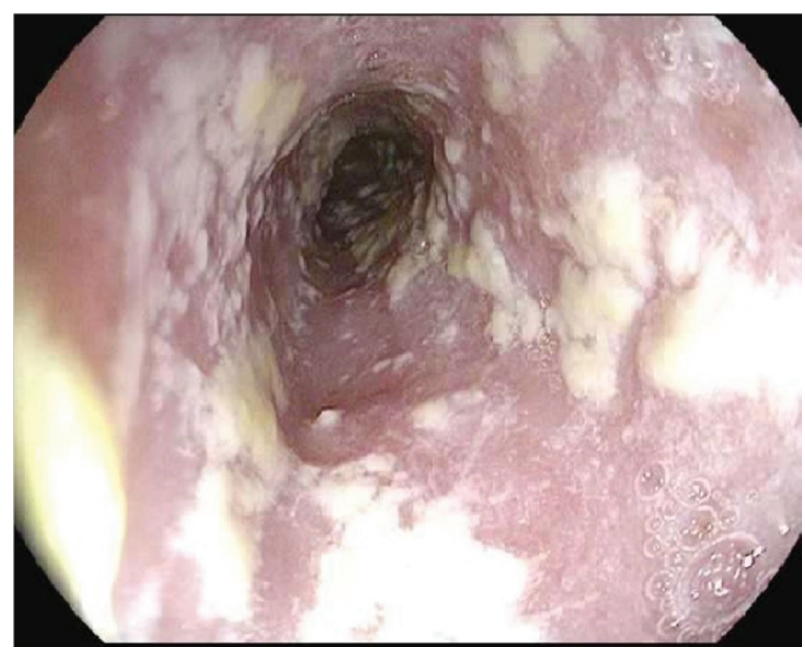

Figure 3. EGD: lower third of the esophagus.

fection and no evidence of opportunistic infections to suggest immunodeficiency $[2,3]$.

The prevalence of EC as observed by Choi et al $(0.32 \%$, $281 / 88,125$ ) [4] is lower compared to data reported by Underwood et al $(0.71 \%, 18 / 2,527)[5]$ and by Naito et al $(1.17 \%$, $41 / 3,501$ ) [6]. Since the early 1980s, most studies of EC have involved HIV-infected patients. However, the frequent use of screening endoscopy has led to more frequent diagnosis of EC in healthy individuals $[7,8]$.

In terms of pathogenesis, the development of EC is described as a two-step process consisting of colonization of the esophagus and subsequent invasion of the epithelial layer [9, 10]. It is already well accepted that Candida colonizes the esophagus of $20 \%$ of healthy adults. Once colonization has been established, impaired cellular immunity permits invasion of the epithelial layer, such as HIV infection [9, 10]. Although EC is well known to occur in immunocompromised hosts, it has only rarely been reported in apparently immunocompetent persons. When it is present in such hosts, it is presumed to accompany a series of predisposing medical conditions $[5,11]$. However, in clinical practice, such underlying conditions are hard to be identified for every case.

A recent study investigated the prevalence and clinical presentation of EC along with the presence and impact of potent predisposing factors in the immunocompetent patient. During the study period, among 30,052 patients who underwent upper gastrointestinal endoscopy, $55(0.18 \%)$ were diagnosed as having EC. Age distribution revealed a skewed distribution to the right, indicating that $\mathrm{EC}$ in the immunocompetent patient follows an age-related pattern, revealing that EC is much more common in patients above the age of 50 [12]. Another retrospective case-control study of EC in individuals older than 65 years found this condition to be a strong predictor of poor survival in older people irrespective of their functional status [13].

Gastric acid suppression is considered to be an important risk factor for the development of EC. Proton pump inhibitors $[14,15], \mathrm{H}_{2}$-receptors antagonists and prior vagotomy produce hypochlorhydria [16], which alters the colonization of 
the stomach by oral cavity bacteria and yeast and is thought to increase the risk of infectious esophagitis. The association of acid suppression therapy with EC was also shown by a recent retrospective case-control study that included 250 patients [17]. Another retrospective study found acid-suppressive therapy with PPIs and $\mathrm{H}_{2}$-receptor antagonists as the most common predisposing risk factor for EC [5].

The use of antibiotics and both systemic and inhaled corticosteroids has also been associated with the development of EC. Antibiotics may predispose immunocompetent patients to fungal infection by allowing overgrowth and colonization of the species. Systematically administered corticosteroids predispose to infection by suppressing both lymphocyte and granulocyte function [2]. Inhaled corticosteroids may be deposited in the esophagus after swallowing and facilitate subsequent colonization and infection with Candida $[18,19]$. EC may also occur in patients with chronic debilitating disease who have received broad-spectrum antibiotics, steroids and immunosuppressants $[20,21]$.

$\mathrm{DM}$ is an important and well recognized risk factor for EC [22]. The association of DM with EC was further shown by a retrospective study including 51 patients [23]. The same study described uncontrolled DM, along with carcinoma, corticosteroid and antibiotic use as the most common risk factors for EC [23]. The association of DM with EC is often described when it is poorly controlled [23, 24]. Another retrospective study from Korea involving $281 \mathrm{HIV}$-negative patients with EC found that there was no underlying disease in $69.8 \%$ of patients, while DM (8.2\%) and malignancy (7.8\%) were the two most common concomitant diseases [4].

In terms of symptomatology, patients with EC may remain asymptomatic or may present with one of the classic symptoms of infectious esophagitis, such as dysphagia, odynophagia and chest pain. As reported by Naito et al in 1988, 3,501 patients undergoing routine EGD in their study, 41 were found to have EC, and two-thirds of those patients had no esophageal symptoms [6]. For EC, EGD with brushings or biopsy remains the most sensitive and specific method of diagnosis $[2,6,8]$.

EC requires systemic antifungal therapy [25], and it should never be managed with local agents [26]. Oral fluconazole is generally recommended for the treatment of EC due to its excellent efficacy, ease of administration and low cost [27]. For patients with documented EC that is refractory to fluconazole after 1 week of treatment, voriconazole and posaconazole are alternative choices in the outpatient setting. Itraconazole oral suspension is also effective, but causes more nausea. In patients in whom intravenous therapy is necessary, an echinocandin can be used [27]. In three randomized trials, fluconazole was superior to ketoconazole, flucytosine and itraconazole for the treatment of EC $[28,29]$. In a number of trials and smaller studies, the effectiveness of fluconazole therapy has ranged from $80 \%$ to $90 \%[26,30,31]$.

In conclusion, EC is a rare condition among immunocompetent patients. Such patients may lack clinical symptoms and/ or predisposing factors. However, underlying conditions occur more frequently in the symptomatic patient. Endoscopic findings correlate neither with clinical manifestations nor with the presence of risk factors [12]. The lack of correlation between clinic symptoms and endoscopic findings was also shown by another retrospective study [23].

In our patient the most likely predisposing factor for EC was uncontrolled DM, and although it is hard to attribute all her symptoms to this etiology, it was an important consideration given her significant symptomatic improvement following therapy with fluconazole. She was educated on the need for a more stringent control of her DM to prevent long-term complications and end-organ damage.

We conclude that although EC in the majority of cases is an incidental finding during EGD, its detection and treatment can provide significant symptomatic relief to patients. It should be considered in the presence of esophageal symptoms of dysphagia, odynophagia and retrosternal discomfort, and this is a particularly pertinent consideration in patients with an underlying predisposing condition like HIV, uncontrolled DM, malignancy, corticosteroid and/or PPI use.

\section{Conflict of Interest}

None.

\section{References}

1. Laine L, Bonacini M. Esophageal disease in human immunodeficiency virus infection. Arch Intern Med. 1994;154(14):1577-1582.

2. Baehr PH, McDonald GB. Esophageal infections: risk factors, presentation, diagnosis, and treatment. Gastroenterology. 1994;106(2):509-532.

3. Thapa BR, Kumar L. Candida esophagitis after antibiotic use. Indian J Pediatr. 1989;56(2):296-299.

4. Choi JH, Lee CG, Lim YJ, Kang HW, Lim CY, Choi JS. Prevalence and risk factors of esophageal candidiasis in healthy individuals: a single center experience in Korea. Yonsei Med J. 2013;54(1):160-165.

5. Underwood JA, Williams JW, Keate RF. Clinical findings and risk factors for Candida esophagitis in outpatients. Dis Esophagus. 2003;16(2):66-69.

6. Naito Y, Yoshikawa T, Oyamada H, Tainaka K, Morita Y, Kogawa T, Sugino S, et al. Esophageal candidiasis. Gastroenterol Jpn. 1988;23(4):363-370.

7. Phaosawasdi K, Rice P, Lee B. Primary and secondary Candida esophagitis. IMJ Ill Med J. 1986;169(6):361365.

8. Mathieson R, Dutta SK. Candida esophagitis. Dig Dis Sci. 1983;28(4):365-370.

9. Hoshika K, Iida M, Mine H. Esophageal Candida infection and adherence mechanisms in the nonimmunocompromised rabbit. J Gastroenterol. 1996;31(3):307-313.

10. Vermeersch B, Rysselaere M, Dekeyser K, Rasquin K, De Vos M, Elewaut A, Barbier F. Fungal colonization of the esophagus. Am J Gastroenterol. 1989;84(9):1079-1083.

11. Wilcox CM, Karowe MW. Esophageal infections: etiology, diagnosis, and management. Gastroenterologist. 1994;2(3):188-206.

12. Mimidis K, Papadopoulos V, Margaritis V, Thomopoulos K, Gatopoulou A, Nikolopoulou V, Kartalis G. Pre- 
disposing factors and clinical symptoms in HIV-negative patients with Candida oesophagitis: are they always present? Int J Clin Pract. 2005;59(2):210-213.

13. Weerasuriya N, Snape J. A study of candida esophagitis in elderly patients attending a district general hospital in the UK. Dis Esophagus. 2006;19(3):189-192.

14. Karmeli Y, Stalnikowitz R, Eliakim R, Rahav G. Conventional dose of omeprazole alters gastric flora. Dig Dis Sci. 1995;40(9):2070-2073.

15. Larner AJ, Lendrum R. Oesophageal candidiasis after omeprazole therapy. Gut. 1992;33(6):860-861.

16. Brooks JR, Smith HF, Pease FB, Jr. Bacteriology of the stomach immediately following vagotomy: the growth of Candida albicans. Ann Surg. 1974;179(6):859-862.

17. Kim KY, Jang JY, Kim JW, Shim JJ, Lee CK, Dong SH, Kim HJ, et al. Acid suppression therapy as a risk factor for Candida esophagitis. Dig Dis Sci. 2013;58(5):12821286.

18. Kanda N, Yasuba H, Takahashi T, Mizuhara Y, Yamazaki S, Imada Y, Izumi Y, et al. Prevalence of esophageal candidiasis among patients treated with inhaled fluticasone propionate. Am J Gastroenterol. 2003;98(10):2146-2148.

19. Kesten S, Hyland RH, Pruzanski WR, Kortan PP. Esophageal candidiasis associated with beclomethasone dipropionate aerosol therapy. Drug Intell Clin Pharm. 1988;22(78):568-569.

20. Andersen LI, Frederiksen HJ, Appleyard M. Prevalence of esophageal Candida colonization in a Danish population: special reference to esophageal symptoms, benign esophageal disorders, and pulmonary disease. J Infect Dis. 1992;165(2):389-392.

21. Alexander JA, Brouillette DE, Chien MC, Yoo YK, Tarter RE, Gavaler JS, Van Thiel DH. Infectious esophagitis following liver and renal transplantation. Dig Dis Sci. 1988;33(9):1121-1126.

22. Bartholomew GA, Rodu B, Bell DS. Oral candidiasis in patients with diabetes mellitus: a thorough analysis. Diabetes Care. 1987;10(5):607-612.

23. Yakoob J, Jafri W, Abid S, Jafri N, Islam M, Hamid $\mathrm{S}$, Shah HA, et al. Candida esophagitis: risk factors in
non-HIV population in Pakistan. World J Gastroenterol. 2003;9(10):2328-2331.

24. Bodey G. Hematogenous and major organ candidiasis. In: Bodey GP (ed) Candidiasis: Pahtogenesis, diagnosis and Treatment: Raven Press, New York; 1993.

25. Panel on Opportunistic Infections in HIV-Infected Adults and Adolescents. Guidelines for the prevention and treatment of opportunistic infections in HIV-infected adults and adolescents: Recommendations from the Centers for Disease Control and Prevention, the National Institutes of Health, and the HIV Medicine Association of the Infectious Diseases Society of America. . 2015 [cited 4/20/2015]; Available from: http://aidsinfo.nih.gov/contentfiles/lvguidelines/adult_oi.pdf.

26. Darouiche RO. Oropharyngeal and esophageal candidiasis in immunocompromised patients: treatment issues. Clin Infect Dis. 1998;26(2):259-272; quiz 273-254.

27. Kauffman CA. Treatment of oropharyngeal and esophageal candidiasis. In: UpToDate. 2015 3/26/2015 [cited 2015 6/10/2015]; Available from: www.uptodate.com.

28. Barbaro G, Barbarini G, Calderon W, Grisorio B, Alcini P, Di Lorenzo G. Fluconazole versus itraconazole for candida esophagitis in acquired immunodeficiency syndrome. Candida Esophagitis. Gastroenterology. 1996;111(5):1169-1177.

29. Laine L, Dretler RH, Conteas CN, Tuazon C, Koster FM, Sattler F, Squires K, et al. Fluconazole compared with ketoconazole for the treatment of Candida esophagitis in AIDS. A randomized trial. Ann Intern Med. 1992;117(8):655-660.

30. Krause DS, Simjee AE, van Rensburg C, Viljoen J, Walsh TJ, Goldstein BP, Wible M, et al. A randomized, double-blind trial of anidulafungin versus fluconazole for the treatment of esophageal candidiasis. Clin Infect Dis. 2004;39(6):770-775.

31. Barbaro G, Barbarini G, Di Lorenzo G. Fluconazole compared with itraconazole in the treatment of esophageal candidiasis in AIDS patients: a double-blind, randomized, controlled clinical study. Scand J Infect Dis. $1995 ; 27(6): 613-617$. 